Pesq. Vet. Bras. 29(4):286-290, abril 2009

\title{
Relação entre a biometria ultra-sonográfica em modo B do bulbo ocular e os diâmetros fronto occiptal e bizigomático em Canis familiaris ${ }^{1}$
}

INDEX TERMS: B-mode ultrasonography, ocular biometry, bizigomatic diameter, fronto-occiptal diameter, dogs.

RESUMO.- Avaliou-se 31 cães saudáveis, sem raça definida, sendo 10 machos e 21 fêmeas, com 8 meses a 7 anos de idade e peso de1,5-28 kg. Inicialmente foram mensurados os diâmetros fronto-occiptal (DFO) e bizigomático (DBZ) do crânio com o auxílio de um paquímetro. A ultra-sonografia transpalpebral em modo-B foi realizada para mensurar as estruturas do bulbo ocular, conforme se segue: D1- espessura da córnea; D2- distância entre o ponto central da imagem da córnea e a da cápsula anterior do cristalino (câmara anterior); D3- distância entre

\footnotetext{
${ }^{1}$ Recebido em 21 de julho de 2008.

Aceito para publicação em 3 de dezembro de 2008.

${ }^{2}$ Laboratório de Biologia e Medicina de Animais da Amazônia, Medicina Veterinária, Universidade Federal do Pará (UFPA), Rua Hernane Lameira 556, Castanhal, PA 68743-050, Brasil. *Autor para correspondência: shfarha@ufpa.br
}

o ponto central da imagem da córnea e a da cápsula posterior do cristalino; D4- espessura do cristalino, que corresponde a distância entre a imagem da cápsula anterior e a cápsula posterior do cristalino; D5- diâmetro do cristalino, distância entre as imagens dos pólos do cristalino; D6- área do cristalino; D7- câmara vítrea, distância entre a imagem da cápsula posterior do cristalino e a retina; D8- distância entre a cápsula anterior do cristalino e a retina; D9- distância entre a imagem da córnea e a retina. Com exceção da D4, houve efeito dos DFO e DBZ sobre as medidas das estruturas internas do BO. A análise de regressão linear entre as medidas das estruturas do bulbo ocular e os DFO e DBZ foram significativas para D1, D2, D3, D4, D5, D6, D7, D8 e D9.

TERMOS DE INDEXAÇÃO: Ultra-Sonografia em modo-B, biometria ocular, diâmetro fronto-occiptal, diâmetro bizigomático, estruturas do bulbo ocular, cães. 


\section{INTRODUÇÃO}

A ultra-sonografia bidimensional (2D) em modo B é uma importante técnica de diagnóstico por imagem para a oftalmologia, devido a sua capacidade de detectar o contorno e o formato das estruturas do bulbo ocular, mesmo quando ocorre a opacidade dessas (Osuobeni \& Hamidzada 1999, González et al. 2001). A ecobiometria ocular em modo B fornece medidas do comprimento axial do bulbo ocular (BO), que permitem avaliar afecções como: glaucoma, microftalmia, macroftalmia, estafiloma, phthisis bulbi e coloboma (Hernández-Guerra \& López-Murcia 2007).

$\mathrm{O}$ estudo do comprimento axial do BO, por meio da biometria ultra-sonográfica, pode auxiliar no cálculo para obtenção do tamanho do BO protético, quando é necessário a enucleação do mesmo, bem como no cálculo do grau (dioptria) da lente intra-ocular (LIO) em pacientes submetidos a cirurgia de catarata, na qual é realizada a substituição do cristalino por uma prótese (LIO) (González 2001). Essa lente artificial (LIO) pode alcançar um poder dióptrico próximo ou igual de um cristalino emétrope. Outra vantagem da ecobiometria em modo $B$ é que essa possibilita a mensuração do comprimento axial das estruturas internas do BO, tais como: distância entre a córnea e a cápsula anterior da lente, densidade da lente, corpo vítreo (distância entre a cápsula posterior da lente até a retina) e a distância entre a córnea e a retina (Cotrill et al. 1989, González 2001).

O formato e o tamanho do crânio canino variam de acordo com a raça, a idade do animal ou a conformação do indivíduo (Dyce et al. 1997). As variações de crânio em Canis familiaris são expressas pela relação entre os diâmetros fronto-occiptal (DFO) e bizigomático (DBZ) (Dyce et al. 1997), sendo esses classificados em: dolicocéfalos (DFO >DBZ), braquicéfalos (DFO <DBZ) e mesocéfalos (DFO $\approx \mathrm{DBZ}$ ) (Getty 1986). Em um estudo comparando a ecobiometria em modos A e bidimensional B com a paquimetria na avaliação de olhos normais em cadáveres de cães dolicocéfalos e mesocéfalos, demonstrou-se que a ultra-sonografia bidimensional em modo $B$ foi tão eficiente quanto à paquimetria para determinar o comprimento axial do BO. Sendo que o seu valor médio em cães dolicocéfalos era significativamente mais longo que em animais mesocéfalos (Cotrill et al. 1989). Esses resultados demonstram que a conformação do crânio pode influenciar no comprimento axial do $\mathrm{BO}$ e de suas estruturas internas, o que dificulta a definição de parâmetros de normalidade ecobiométrica ocular em cães, uma vez que a espécie apresenta grande variação do formato de crânios.

Considerando que o efeito da conformação do crânio sob o comprimento axial e estruturas internas do BO ainda não foi completamente elucidado na espécie canina, o objetivo do presente trabalho foi avaliar a relação e o efeito entre os diâmetros fronto-occiptal (DFO) e bizigomático (DBZ) e as medidas biométricas das estruturas do bulbo ocular de cães, a fim de estabelecer parâmetros confiáveis de normalidade.

\section{MATERIAL E MÉTODOS}

O experimento foi realizado no Laboratório de Biologia e Medicina de Animais Silvestres e Domésticos da Amazônia (BIOMEDAM), Universidade Federal do Pará (UFPA), localizado na cidade de Castanhal (Pará). Foram avaliados 31 cães saudáveis, sem raça definida, sendo 10 machos e 21 fêmeas, com idade de1-7 anos de idade e peso de 1,5-28 kg. Inicialmente foram mensurados os DFO e DBZ do crânio com o auxílio de um paquímetro (Zaas, São Paulo, SP, Brasil) (Fig.1A e 1B).

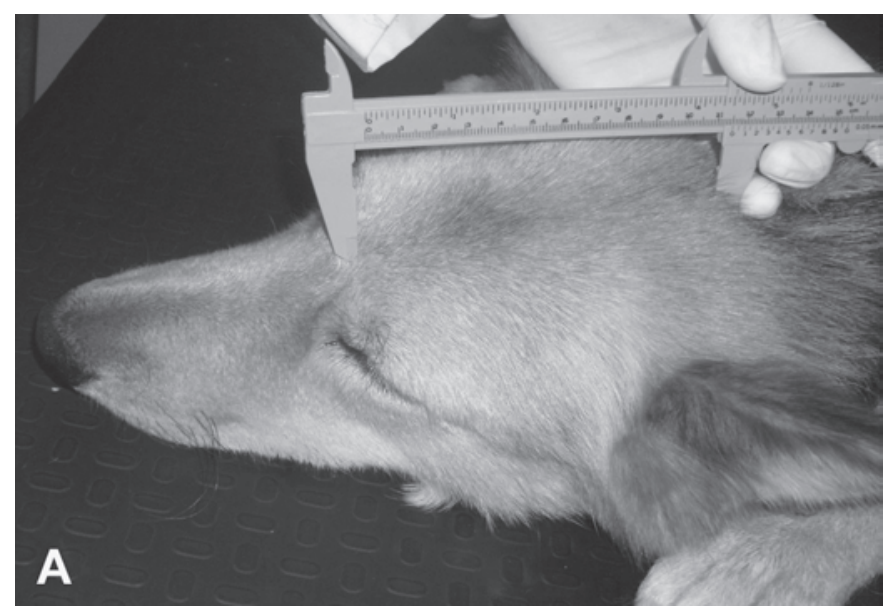

Fig.1. Mensuração com paquímetro dos diâmetros frontoocciptal e bizigomático. (A) Mensuração do diâmetro fronto-occiptal; (B) Mensuração do diâmetro bizigomático.

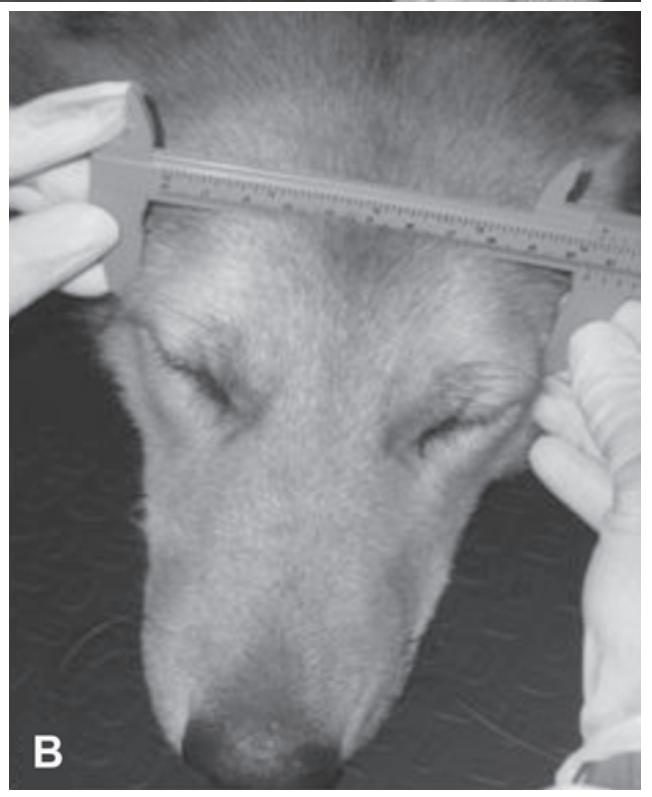

Para a realização do exame ultra-sonográfico, foi conduzida a contenção mecânica dos animais. No experimento, a técnica adotada para realizar o exame ultra-sonográfico do bulbo ocular foi a transpalpebral, na posição sentada ou em decúbito esternal.

As imagens foram obtidas utilizando o ultra-som Philips HDI 4000 (Philips Medical Systems, Bothel, WA, USA), em modo B, com transdutor microconvexo multifreqüêncial de 5-8 $\mathrm{MHz}$. Previamente ao exame, foi aplicado entre o transdutor e as pálpebras, gel ultra-sonográfico hipoalérgico (Aquasonic, Parker Laboratories, INC, Fairfield, New Jersey). Após o exame as pálpebras foram cuidadosamente limpas para a remoção do gel com compressas de algodão (Compressa de Gaze Hidrófila, Cremer, Blumenau, SC, Brasil) umedecidas com solução 


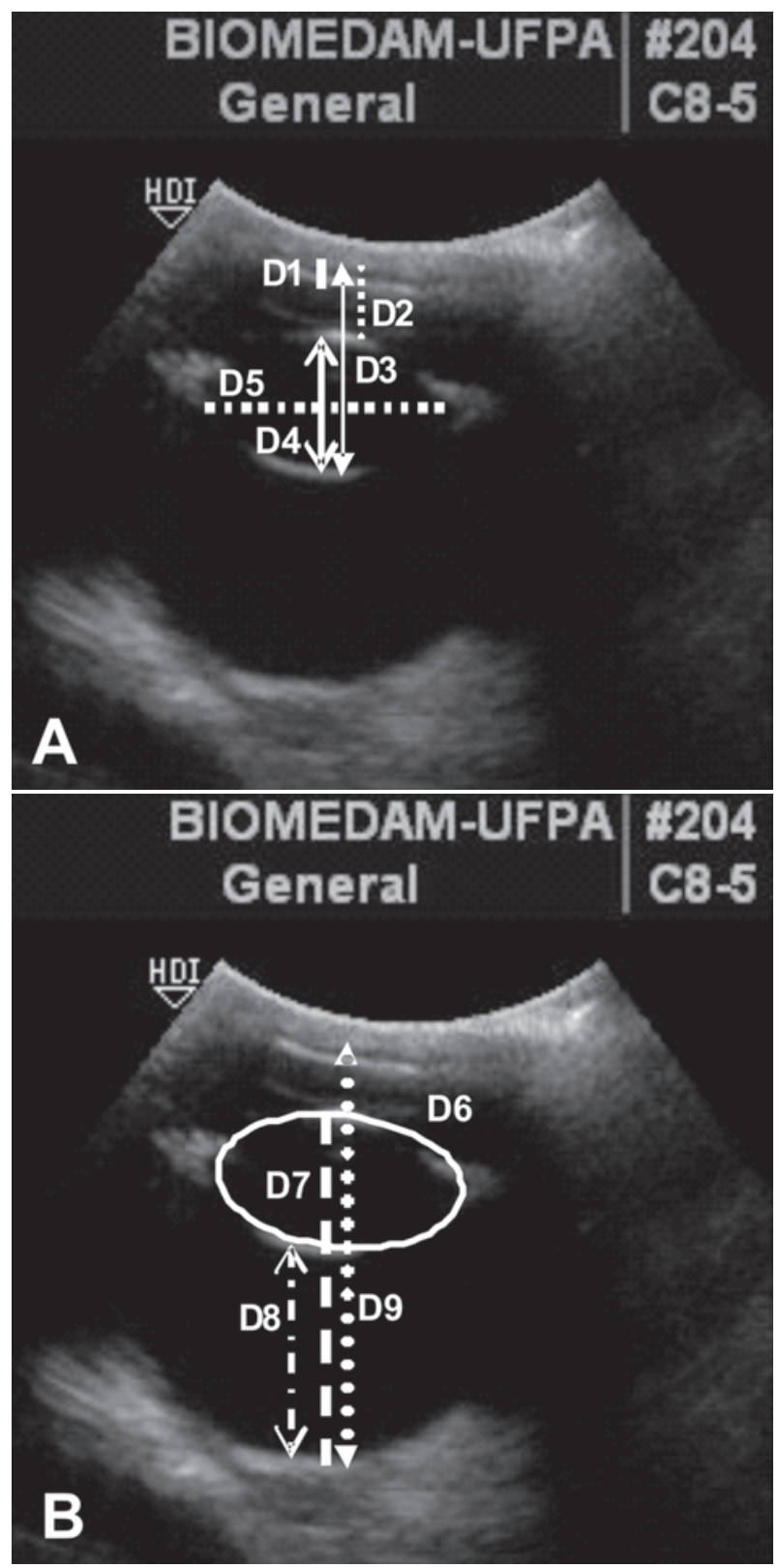

Fig.2. Esquema das mensurações das estruturas internas do globo ocular em Canis familiaris adultos. A) D1 = espessura da córnea; D2 = distância entre o ponto central da imagem da córnea e a da cápsula anterior do cristalino; D3 = distância entre o ponto da imagem da córnea e a da cápsula posterior do cristalino; D4 - espessura do cristalino; D5 = diâmetro do cristalino. B) D6 = área do cristalino; D7 = câmara vítrea; D8 = distância da cápsula anterior do cristalino e retina; D9 = distância entre a imagem da córnea e retina.

fisiológica ( $\mathrm{NaCl}$ 0,9\%, Indufal, Mossoró, RN, Brasil). As medidas obtidas foram as seguintes: D1- espessura da córnea; D2distância entre o ponto central da imagem da córnea e a da cápsula anterior do cristalino (câmara anterior); D3- distância entre o ponto central da imagem da córnea e a da cápsula posterior do cristalino; D4- espessura do cristalino, que corresponde a distância entre a imagem da cápsula anterior e a cápsula posterior do cristalino; D5- diâmetro do cristalino, distância entre as imagens dos pólos do cristalino; D6- área do cristalino; D7- câmara vítrea, distância entre a imagem da cápsula posterior do cristalino e a retina; D8- distância entre a cápsula anterior do cristalino e a retina; D9- distância entre a imagem da córnea e a retina (Fig.2A e 2B). Os dados foram representados em média \pm erro padrão. As médias das estruturas internas do BO direito e esquerdo e entre machos e fêmeas foram comparadas entre si pelo teste $t$ de Student. Para avaliar o efeito DFO e DBZ sobre o diâmetro das estruturas do bulbo ocular foi utilizada a ANOVA, dividindo os animais em três grupos diferentes de DFO e DBZ nas medidas: $5,0-7,5 \mathrm{~cm}$, 8,0-10,0cm, $11,0-13,0 \mathrm{~cm}$ e $5,0-6,5 \mathrm{~cm}, 7,0-10 \mathrm{~cm}, 11,0-12,0 \mathrm{~cm}$, respectivamente. As diferenças estatísticas entre as médias foram determinadas pelo teste de Fisher PLSD. Análises de regressão foram feitas entre as medidas lineares das estruturas do BO com os DFO e DBZ, respectivamente. Todos os testes foram aplicados a $1 \%$ de probabilidade. As análises estatísticas foram realizadas com o software estatístico Stat View (SAS Institute. Inc., Cary, NC, USA).

\section{RESULTADOS}

O total de 62 BO normais foi avaliado, referente aos 31 animais utilizados neste experimento. $\mathrm{O}$ transdutor microconvexo multifreqüêncial de 5-8 MHz possibilitou a realização do exame por via transpalpebral sem almofada de recuo. Não houve diferenças estatísticas entre as medidas biométricas das estruturas do bulbo ocular quando comparados os olhos direito e esquerdo entre machos e fêmeas.

\section{Efeito e relação do DFO sobre as medidas das estru- turas do bulbo ocular}

No Quadro 1 estão demonstradas as análises de variância entre os diferentes grupos de tamanhos do DFO e os parâmetros das estruturas internas do BO. Houve efeito significativo $(P<0,01)$ do DFO sobre as medidas das estruturas do bulbo ocular D1, D2, D3, D5, D6, D7, D8,

Quadro 1. Média \pm EP das estruturas internas $(\mathrm{cm})$ do bulbo ocular de acordo com a categoria de diâmetro fronto-occiptal de cães adultos SRD

\begin{tabular}{ccccc}
\hline \multirow{2}{*}{$\begin{array}{c}\text { Parâmetros } \\
(\mathrm{cm})^{\mathrm{a}}\end{array}$} & $5,0-7,5$ & $8,0-10,0$ & $11,0-13,0$ & $P$ \\
\cline { 2 - 5 } D1 & $0,07 \pm 0,02^{\mathrm{a}}$ & $0,08 \pm 0,002^{\mathrm{b}}$ & $0,10 \pm 0,003^{\mathrm{c}}$ & 0,001 \\
D2 & $0,26 \pm 0,01^{\mathrm{a}}$ & $0,32 \pm 0,007^{\mathrm{b}}$ & $0,39 \pm 0,02^{\mathrm{c}}$ & 0,001 \\
D3 & $0,91 \pm 0,01^{\mathrm{a}}$ & $0,98 \pm 0,01^{\mathrm{b}}$ & $1,15 \pm 0,02^{\mathrm{c}}$ & 0,001 \\
D4 & $0,69 \pm 0,01^{\mathrm{a}}$ & $0,65 \pm 0,01^{\mathrm{a}}$ & $0,72 \pm 0,01^{\mathrm{a}}$ & 0,53 \\
D5 & $1,01 \pm 0,01^{\mathrm{a}}$ & $1,05 \pm 0,02^{\mathrm{a}}$ & $1,15 \pm 0,02^{\mathrm{b}}$ & 0,004 \\
D6 & $0,51 \pm 0,01^{\mathrm{a}}$ & $0,51 \pm 0,01^{\mathrm{a}}$ & $0,61 \pm 0,01^{\mathrm{b}}$ & 0,006 \\
D7 & $0,82 \pm 0,01^{\mathrm{a}}$ & $0,86 \pm 0,01^{\mathrm{a}}$ & $0,97 \pm 0,02^{\mathrm{b}}$ & 0,002 \\
D8 & $1,47 \pm 0,02^{\mathrm{a}}$ & $1,49 \pm 0,01^{\mathrm{a}}$ & $1,66 \pm 0,01^{\mathrm{b}}$ & 0,001 \\
D9 & $1,70 \pm 0,02^{\mathrm{a}}$ & $1,81 \pm 0,03^{\mathrm{b}}$ & $2,06 \pm 0,04^{\mathrm{c}}$ & 0,001
\end{tabular}

a D1= espessura da córnea; D2 = distância entre o ponto central da imagem da córnea e a da cápsula anterior do cristalino; D3 = distância entre o ponto da imagem da córnea e a da cápsula posterior do cristalino; D4 = espessura do cristalino; D5 = diâmetro do cristalino; D6 = área do cristalino; D7 = câmara vítrea; D8 = distância da cápsula anterior do cristalino e retina; D9 = distância entre a imagem da córnea e retina. Letras diferentes indicam diferenças estatísticas entre as médias das estruturas internas do GO quando comparado entre os diferentes grupos $(P<0,01)$. 
Quadro 2. Média \pm EP das estruturas internas do bulbo
ocular de acordo com a categoria de diâmetro bizigomático
de cães adultos SRD

\begin{tabular}{|c|c|c|c|c|}
\hline \multirow{2}{*}{$\begin{array}{l}\text { Parâmetros } \\
(\mathrm{cm})^{\mathrm{a}}\end{array}$} & \multicolumn{4}{|c|}{ Diâmetro bizigomáticol $(\mathrm{cm})$} \\
\hline & $5,0-6,5$ & $7,0-10$ & $11,0-12,0$ & $P$ \\
\hline D1 & $0,08 \pm 0,001^{a}$ & $0,08 \pm 0,003^{b}$ & $0,10 \pm 0,005^{c}$ & 0,001 \\
\hline $\mathrm{D} 2$ & $0,28 \pm 0,01^{a}$ & $0,33 \pm 0,01^{a}$ & $0,38 \pm 0,00^{b}$ & 0,01 \\
\hline D3 & $0,94 \pm 0,01^{a}$ & $0,99 \pm 0,03^{a}$ & $1,14 \pm 0,01^{b}$ & 0,003 \\
\hline D4 & $0,67 \pm 0,008^{a}$ & $0,70 \pm 0,02^{a}$ & $0,71 \pm 0,03^{a}$ & 0,16 \\
\hline D5 & $1,04 \pm 0,01^{a}$ & $1,11 \pm 0,02^{b}$ & $1,10 \pm 0,02^{b}$ & 0,005 \\
\hline D6 & $0,52 \pm 0,01^{a}$ & $0,55 \pm 0,01^{a}$ & $0,60 \pm 0,007^{b}$ & 0,002 \\
\hline D7 & $0,83 \pm 0,01^{a}$ & $0,90 \pm 0,01^{b}$ & $1,00 \pm 0,01^{c}$ & 0,001 \\
\hline D8 & $1,47 \pm 0,01^{a}$ & $1,58 \pm 0,01^{a}$ & $1,68 \pm 0,03^{b}$ & 0,002 \\
\hline D9 & $1,71 \pm 0,02^{a}$ & $1,90 \pm 0,01^{b}$ & $2,14 \pm 0,02^{c}$ & 0,001 \\
\hline
\end{tabular}

a D1 = espessura da córnea; D2 = distância entre o ponto central da imagem da córnea e a da cápsula anterior do cristalino; D3 = distância entre o ponto da imagem da córnea e a da cápsula posterior do cristalino; D4 = espessura do cristalino; D5 =- diâmetro do cristalino, D6 = área do cristalino; D7 = câmara vítrea; D8 = distância da cápsula anterior do cristalino e retina; D9 = distância entre a imagem da córnea e retina. Letras diferentes indicam diferenças estatísticas entre as médias das estruturas internas do GO quando comparado entre os diferentes grupos $(P<0,01)$.

Quadro 3. Análise de regressão linear entre as distâncias das estruturas internas do bulbo ocular e o diâmetro fronto-occiptal em cães adultos SRD $(P<0,01)$

\begin{tabular}{ccc}
\hline Parâmetro $(\mathrm{cm})^{\mathrm{a}}$ & Equação & $\mathrm{R}^{2}$ \\
\hline D1 & D1 $=0,041+0,005$ DFO & 0,76 \\
D2 & D2 $=0,070+0,027$ DFO & 0,90 \\
D3 & D3 $=0,677+0,038$ DFO & 0,84 \\
D4 & D4 $=0,633+0,007$ DFO & 0,52 \\
D5 & D5 $=0,828+0,028$ DFO & 0,64 \\
D6 & D6 $=0,374+0,021$ DFO & 0,92 \\
D7 & D7 $=0,607+0,029$ DFO & 0,82 \\
D8 & D8 $=1,189+0,038$ DFO & 0,85 \\
D9 & D9 $=1,329+0,061$ DFO & 0,82 \\
\hline
\end{tabular}

a D1= espessura da córnea; D2 = distância entre o ponto central da imagem da córnea e a da cápsula anterior do cristalino; D3 = distância entre o ponto da imagem da córnea e a da cápsula posterior do cristalino; D4 = espessura do cristalino; D5 =- diâmetro do cristalino, D6 = área do cristalino; D7 = câmara vítrea; $D 8$ = distância da cápsula anterior do cristalino e retina; D9 = distância entre a imagem da córnea e retina. $R^{2}=$ Coeficiente de determinação.

D9. A D4 não variou de acordo com tamanho do DFO. As médias das estruturas do bulbo ocular D1, D2, D3 e D9 foram diferentes entre os três grupos de tamanhos do DFO. As médias D5, D6, D7 e D8 foram estatisticamente diferentes quando comparados os animais com DFO entre 5$10 \mathrm{~cm}$ e $11-13 \mathrm{~cm}(P<0,01)$. No Quadro 3 estão demonstrados as análises de regressão entre a biometria das estruturas do bulbo ocular e os DFO, sendo que todos foram significativos para o intercepto $(P<0,05)$ e coeficientes de regressão $(P<0,01)$.

\section{Efeito e relação do DBZ sobre as medidas das estru- turas do bulbo ocular}

No Quadro 2 estão demonstradas as análises de variância entre os diferentes grupos de tamanhos do DBZ e os parâmetros das estruturas internas do BO. Houve
Quadro 4. Análise de regressão linear entre as distâncias das estruturas internas do bulbo ocular e o diâmetro bizigomático em cães adultos SRD $(P<0,01)$

\begin{tabular}{ccc}
\hline Parâmetro $(\mathrm{cm})^{\mathrm{a}}$ & Equação & $\mathrm{R}^{2}$ \\
\hline D1 & D1 $=0,054+0,004 \mathrm{DBZ}$ & 0,86 \\
D2 & D2 $=0,169+0,02 \mathrm{DBZ}$ & 0,92 \\
D3 & D3 $=0,688+0,04 \mathrm{DBZ}$ & 0,98 \\
D4 & D4 $=0,644+0,006 \mathrm{DBZ}$ & 0,80 \\
D5 & D5 $=0,806+0,039 \mathrm{DBZ}$ & 0,96 \\
D6 & D6 $=0,405+0,019 \mathrm{DBZ}$ & 0,89 \\
D7 & D7 $=0,672+0,027 \mathrm{DBZ}$ & 0,71 \\
D8 & D8 $=1,232+0,041 \mathrm{DBZ}$ & 0,88 \\
D9 & D9 $=1,245+0,081 \mathrm{DBZ}$ & 0,91 \\
\hline
\end{tabular}

a D1= espessura da córnea; D2 = distância entre o ponto central da imagem da córnea e a da cápsula anterior do cristalino; D3 = distância entre o ponto da imagem da córnea e a da cápsula posterior do cristalino; D4 = espessura do cristalino; D5 = diâmetro do cristalino, D6 = área do cristalino; D7 = câmara vítrea; D8 = distância da cápsula anterior do cristalino e retina; D9 = distância entre a imagem da córnea e retina. $R^{2}$ : Coeficiente de determinação.

efeito significativo $(P<0,01)$ do DBZ sobre as medidas das estruturas do bulbo ocular: D1, D2, D3, D5, D6, D7, D8, D9. A D4 não variou de acordo com o DBZ. As médias D1, D2, D3, D7 e D9 foram diferentes entre os três grupos do DBZ $(P<0,01)$. Na D5 houve diferença estatística quando comparados os animais com DBZ entre 5$6,5 \mathrm{~cm}$ e $7,0-12,0 \mathrm{~cm}(P<0,01)$, enquanto que a D6 e D8 foram estatisticamente diferentes entre grupos de animais com DBZ entre $5-10 \mathrm{~cm}$ e $11-12 \mathrm{~cm}(P<0,01)$. No Quadro 4 estão demonstradas as análises de regressão entre a biometria das estruturas do bulbo ocular e o DBZ, sendo que todos foram significativos para o intercepto $(P<0,05)$ e coeficiente de regressão $(P<0,01)$.

\section{DISCUSSÃO}

A utilização do transdutor microconvexo multifreqüêncial de 5-8 MHz, sem almofada de recuo, forneceu medidas fidedignas das estruturas do bulbo ocular, com o emprego da técnica transpalpebral, ou seja, com as pálpebras fechadas. As vantagens dos transdutores microconvexos em relação a transdutores lineares foram: a fácil execução de manobras em busca de uma boa imagem ultrasonográfica e o menor contato com a pele, proporcionado pela curva do transdutor, obtendo imagens por uma pequena janela acústica (King 2004). A técnica transpalpebral proporcionou um menor incômodo para o paciente, não havendo a necessidade da utilização de um anestésico tópico, também evitando o surgimento de irritações e lesões mecânicas em decorrência do manuseio do transdutor sobre a córnea. A limpeza das pálpebras, no término do exame ultra-sonográfico, com solução fisiológica foi realizada de acordo com o sugerido por Nyland \& Mattoon (2005), evitando que o gel atingisse a córnea e a conjuntiva e permanecessem por um tempo prolongado causando possíveis lesões irritativas.

No presente trabalho foi adotado como parâmetro as medidas do formato do crânio, como os DFO e DBZ para comparar com as médias das estruturas do bulbo ocular 
entre os animais com diferentes conformações do crânio. O peso tem sido utilizado como parâmetro para comparar as médias D1, D2, D3 e D4 em cães de diferentes portes (Sampaio et al. 2002). No entanto, o peso pode variar de acordo com o estado físico do indivíduo, não sendo, portanto, confiável para estimar padrão de normalidade da ecobiometria ocular, uma vez que os diâmetros das estruturas do bulbo ocular não sofrem modificações com o estado físico do animal.

Segundo as análises estatísticas, não houve diferença entre as estruturas do bulbo ocular direito e esquerdo, dessa forma o olho normal pode ser parâmetro confiável para estabelecer o BO esquemático para o olho lesionado ou enucleado. No entanto, em casos de trauma, enucleação ou má formação congênita nos dois olhos, as mensurações dos DFO e DBZ podem auxiliar no cálculo dos bulbos oculares protéticos ou esquemáticos, considerando que foi demonstrado que as medidas D1, D2, D3, D5, D6, D7, D8 e D9 sofrem influência dos aumentos dos DFO e DBZ. No tocante a D4, o efeito do DFO e do DBZ não foi significativo. Esse resultado pode ser explicado pelo mecanismo de acomodação do cristalino, que tem a capacidade de mudar o seu contorno de acordo com o ponto focal no momento do exame (Gonçalves et al. 2000), assim alterando os resultados biométricos do mesmo. Em humanos, é fácil obter com precisão o cálculo da espessura do cristalino, pois o paciente é orientado para realizar manobras de movimentação dos olhos em direções variadas, tais como médio lateral e crânio caudal e não realizar movimentos de piscar, acomodando desta forma o cristalino no $\mathrm{GO}$, esses tipos de manobras tornam-se úteis nos cálculos biométricos de D4, D5, D6, D7 E D8 (Figueirêdo \& Teixeira 2007). No entanto, as relações lineares entre a D4 e os DBZ e DFO foram significativas, sendo que o DBZ apresentou o modelo mais confiável $\left(R^{2}=0,80\right)$, que pode estar relacionado ao fato de que as duas estruturas estão no mesmo plano de secção em relação ao crânio. As análises de regressão são importantes para estimar o olho esquemático e compará-lo com o olho real do cão, podendo ser úteis na prática clínica para avaliar doenças que alteram o comprimento axial tanto do GO quanto de suas estruturas internas.

\section{CONCLUSÕES}

Conforme os dados obtidos neste estudo, foi demonstrado que D1, D2, D3, D5, D6, D7, D8, D9 são relacionadas pelo tipo de crânio canino.

Devido às mudanças da acomodação do cristalino no ato do exame, não foi possível estabelecer o efeito da conformação do crânio sob a D4.

A análise da conformação do crânio em relação à ecobiometria das estruturas internas do BO são parâmetros confiáveis, permitindo avaliar patologias que alteram a biometria do $\mathrm{BO}$, bem como pode auxiliar no estudo de olho esquemático canino e na escolha da melhor fórmula para o cálculo da lente intra-ocular.

\section{REFERÊNCIAS}

Cottrill N.B., Banks W.J. \& Pechman R.D. 1989. Ultrasonographic and biometric evaluation of the eye and orbit of dogs. Am. J. Vet. Res. 50:898-903.

Dyce K.M., Sack W.O. \& Wensing C.J.G. 2004. Órgãos do sentido, p.359-384. In: Dyce K.M., Sack W.O. \& Wensing C.J.G. (ed.), Tratado de Anatomia Veterinária. $3^{a}$ ed. Elsevier, Rio de Janeiro. 165p.

Figueirêdo S.S. \& Teixeira K. 2007. Olho e órbita, p.71-107. In: Sousa L.R.M.F., Nicola H. \& Szejnfeld J. (ed.), Ultra-sonografia de Órgãos e Estruturas Superficiais. Roca, São Paulo.

Getty R.1986. Osteologia do carnívoro, p.1377-1391. In: Getty R. (ed.), Anatomia dos Animais Domésticos. Vol.2. $5^{\mathrm{a}}$ ed. Guanabara Koogan, Rio de Janeiro.

Gonçalves G.F., Pippi L.N., Raiser G.A., Mazzanti A., Oliveira de T.S., Neves J.P., Leotte A.M. \& Hintz C.W. 2000. Biometria ultra-sonográfica bidimensional em tempo real do globo ocular de cães. Ciência Rural 30:417-420.

González E.M., Rodriguez A. \& García I. 2001. Review of ocular ultrasonography. Vet. Radiol. Ultrasound 42:485-495.

Hernández-Guerra A.M. \& López-Murcia M.M. 2007. Ocular biometry in the adult anesthetized ferret (Mustela putorius furo). Vet. Ophthalmol. 10(1):50-52.

Nyland T.G. \& Mattoon J.S. 2005. Olho, p.315-334. In: Nyland T.G. \& Mattoon J.S. (ed.), Ultra-som Diagnóstico em Pequenos Animais. $2^{a}$ ed. Roca, São Paulo.

Osuobeni E.P. \& Hamidzada W.A. 1999. Agreement between A-mode and B-mode ultrasonography in the measurement of ocular distances. Vet. Radiol. Ultrasound 40:502-507.

Sampaio G.R., Ranzani J.J.T. \& Schellini S.A. 2002. Sexo, peso e conformação anatômica do olho sobre cáculode poder dióptrico de lentes intra-oculares no cão. Ciência Rural 32:263-268. 